\title{
BICYCLE WHEEL WOBBLE A Case Study in Dynamics
}

\author{
John V. Ringwood and Ruijuan Feng \\ Dept. of Electronic Engineering \\ NUI Maynooth \\ County Kildare, Ireland \\ john.ringwood@eeng.nuim.ie, ruijuan.feng@nuim.ie
}

Keywords: Bicycle, dynamics, wheel wobble, instability.

\begin{abstract}
This paper examines reasons why wheel wobble occurs in common production bicycles. In particular, the effects of frame size, rider position and riding style are examined with reference to a range of mathematical models of bicycles which are available in the published literature. Much of the motivation for this work comes from the personal cycling experience of one of the authors and the difficulty in resolving the true cause of wheel wobble from the wide range of advice offered of a variety of cycling experts. It is hoped that recourse to a mathematical analysis will give objective direction as to how wheel wobble can be alleviated through rider intervention.
\end{abstract}

\section{INTRODUCTION}

Since 1869 (Rankine, 1869), engineers have been intrigued by the dynamics of the simple bicycle. In recent years, there has been somewhat of a resurgence of interest with the appearance of a number of excellent review papers, such as (Astrom et al., 2005; Limebeer and Sharp, 2006), with reference to almost 170 technical works on the subject. From a dynamics perspective, the study of the bicycle is attractive for many reasons:

- The bicycle is ubiquitous in our lives as a device for commuting, recreation and sport,

- It permits various levels of complexity of analysis, from an interesting lumped-parameter system with non-minimum phase characteristics to a complex system described by a distributedparameter model,

- Since bicycles are relatively easy to construct, they can be used as mechanical engineering testbeds, with a range of configurations limited only by imagination (Klein, 1989), and

- With the drive towards decreased weight and improved performance, commercial developers in bicycle and motorsport have a great interest in the dynamics of single-track vehicles (Beghi and Frezza, 2006; Hauser and Saccon, 2006).

The motivation for the current study comes from the modest, but important, aspiration of trying to stay upright on a road (racing) bicycle during high-speed descents. While it might be true to say that the aspirant in question (one of the authors on this paper!) is not the most accomplished/confident bike rider, some contribution by the bicycle dynamics to the rider's problem is likely, since:

- The author in question has consistently experienced speed wobble (sometimes called 'shimmy' (Brandt, 2005)) with two well-known production bicycles, but not with a third, and

- The bicycles which exhibited wheel wobble have been successfully ridden by many other cyclists (including Lance Armstrong in his 2003 Tour de France success) with no evidence of wheel wobble.

Wheel wobble is a spontaneous steering oscillation of the front wheel, usually building as the speed approaches a certain threshold. Many theories and myths exist in relation to wheel wobble, including:

1. Wheel wobble is a function of the natural bicycle dynamics, not rider induced i.e. it is inherent to the geometry and elasticity of the bicycle frame (Brandt, 2005).

2. The common rider response of gripping the handlebars tighter only serves to increase the oscillation.

3. Shimmy can be minimised by keeping the knee against the crossbar (increases damping). 
4. Shimmy is less prevalent in bicycles which have a longer trail (see model in Section 2).

5. Shimmy is more likely in bicycles with longer frames and higher saddles.

6. Weight distribution has no effect on shimmy although where that weight contacts the frame does (Brandt, 2005).

7. Shimmy is due to loose bearings or eccentrics in the rotational masses (most common reason given, but refuted my many experts).

It is also the experience of the author that shimmy tends to accompanying braking - release of brakes appears to diminish the wobble oscillation amplitude or remove it altogether.

The objective of this study is to examine the range of possible reasons from a pragmatic dynamical systems perspective to see if the root cause of wheel wobble can be identified and to look for any rider interventions (such as weight distribution, braking protocol, etc) which may help to alleviate the symptom.

\section{BICYCLE MODEL}

The model used in this study is based on that presented in (Astrom et al., 2005). Assumptions under which the model is developed include:

- The bicycle consists of four rigid parts: Frame (including rider), front fork with handlebars and two wheels.

- The influence of other moving parts, such as pedals, chain and brakes on the dynamics is neglected.

- The forward velocity of the bicycle, $V$, is constant. Figs. 1 and 2 show the representations of the parameters and variables of the system (respectively), with:

$\mathbf{O}$ being the centre of mass (including rider)

$c$ is known as the trail

$\lambda$ is the head angle

$\phi$ is the lean angle

$\delta$ is the steering angle

The front fork is key to the ability of a bicycle to self-stabilize and the key relationship is that between $\phi$ and $\delta$. For small angles, the front fork roll angle is:

$$
\phi_{f}=\phi-\delta \cos (\lambda)
$$

and the effective front fork steering angle is:

$$
\delta_{f}=\delta \sin (\lambda)
$$

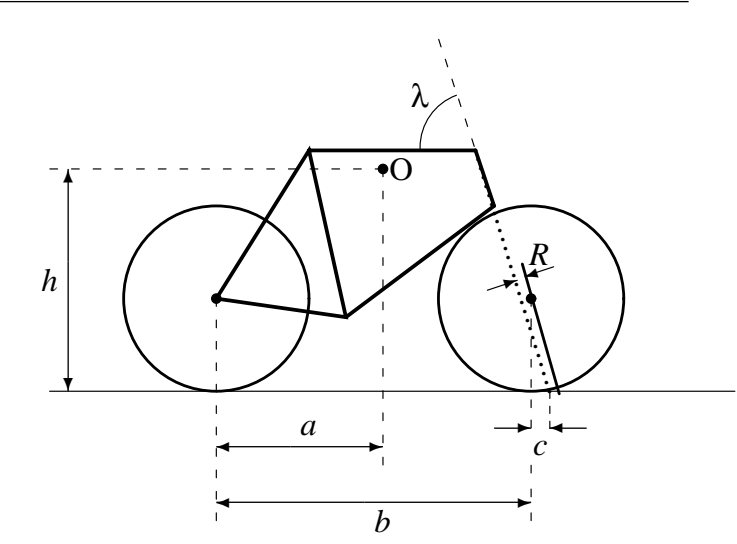

Figure 1: Bicycle parameters.

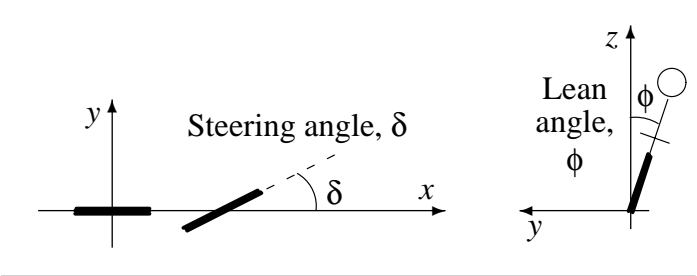

Figure 2: Bicycle variables.

A static torque balance is used to model the front fork, neglecting dynamics and gyroscopic effects. If $N_{f}$ and $F_{f}$ are the vertical and horizontal forces acting on the front wheel at the ground contact, then:

$$
N_{f}=a \mathrm{mg} / \mathrm{b}
$$

where $m$ is the combined mass of rider and frame, and

$$
F_{f}=a m V^{2} \delta_{f} / b^{2}=\frac{a m V^{2} \sin (\lambda)}{b^{2}} \delta
$$

The static torque balance for the front fork, assuming negligible mass of the front fork assembly, is:

$$
T-\left(F_{f}+N_{f} \phi_{f}\right) c \sin (\lambda)
$$

where $\mathrm{T}$ is the external torque applied to the handlebar. Using (1), (2), (3) and (4), (5) reduces to:

$$
\begin{gathered}
T-\frac{a c m g \sin (\lambda)}{b} \phi- \\
\frac{a c m \sin (\lambda)}{b^{2}}\left(V^{2} \sin (\lambda)-b g \cos (\lambda)\right) \delta=0
\end{gathered}
$$

where $g$ is the acceleration due to gravity. The torque balance can be rewritten as:

$$
\delta=k_{1}(V) T-k_{2}(V) \phi
$$

which demonstrates negative feedback stabilization) between $\phi$ and $\delta$ if $k_{2}$ is $+v e$, or

$$
V>\sqrt{b g \cot (\lambda)}
$$


where:

$$
k_{1}(V)=\frac{b^{2}}{\left(V^{2} \sin (\lambda)-b g \cos (\lambda)\right) m a c \sin (\lambda)}
$$

and

$$
k_{2}(V)=\frac{b g}{V^{2} \sin (\lambda)-b g \cos (\lambda)}
$$

Note that the center of mass of the frame is shifted when the front wheel is turned, giving the torque:

$$
T_{\delta}=-\frac{m g a c \sin (\lambda)}{b} \delta
$$

An overall angular momentum balance for the frame can now be constructed as:

$J \frac{d^{2} \phi}{d t^{2}}-m g h \phi=\frac{D V \sin (\lambda)}{b} \frac{d \delta}{d t}+\frac{m\left(V^{2} h-a c g\right) \sin (\lambda)}{b} \delta$

where $J$ is the moment of inertia of the bicycle with respect to the $x$-axis and $D$ is the inertia product with respect to the $x-z$ axes (see Figs.1 and 2). Finally, inserting (6) into the momentum balance gives:

$$
\begin{array}{r}
J \frac{d^{2} \phi}{d t^{2}}+\frac{D V g}{V^{2} \sin (\lambda)-b g \cos (\lambda)} \frac{d \phi}{d t} \\
+\frac{m g^{2}(b h \cos (\lambda)-a \operatorname{csin}(\lambda))}{V^{2} \sin (\lambda)-b g \cos (\lambda)} \phi \\
=\frac{D V b}{a c m\left(V^{2} \sin (\lambda)-b g \cos (\lambda)\right)} \frac{d T}{d t} \\
+\frac{b\left(V^{2} h-a \cos \right)}{a c\left(V^{2} \sin (\lambda)-b g \cos (\lambda)\right)} T
\end{array}
$$

The system poles can be evaluated as:

$$
p_{1,2}=\frac{\frac{-D V g}{K} \pm \sqrt{\frac{d^{2} V^{2} g^{2}}{K_{1}^{2}}-\frac{4 J m g^{2}\left(K_{2}\right)}{K_{1}}}}{2 J}
$$

or

$$
p_{1,2}=\frac{-m a h V g \pm \sqrt{(m a h V g)^{2}-4 m^{2} g^{2} h^{2} K_{1}\left(K_{2}\right)}}{2 m h^{2} K_{1}}
$$

where

$$
K_{1}=V^{2} \sin (\lambda)-b g \cos (\lambda)
$$

and

$$
K_{2}=b h \cos (\lambda)-\operatorname{acsin}(\lambda)
$$

\section{MODEL PARAMETERIZATION}

In this section, the model parameters will be determined for a (2003) Trek 5200. The manufacturer provides a table of some of the critical model dimensions, as they vary with frame size, as: We can provide a
Table 1: Variation in bicycle parameters with frame size.

\begin{tabular}{|c|c|c|c|}
\hline Frame size & $\mathrm{b}(\mathrm{m})$ & $\mathrm{R}$ & $\lambda(\mathrm{deg})$ \\
\hline 50 & 0.979 & 0.0045 & 72 \\
\hline 52 & 0.981 & 0.0045 & 72.5 \\
\hline 54 & 0.985 & 0.0045 & 73 \\
\hline 56 & 0.996 & 0.0045 & 73.8 \\
\hline 58 & 0.996 & 0.0045 & 73.8 \\
\hline 60 & 1.004 & 0.0045 & 74 \\
\hline 62 & 1.008 & 0.0045 & 74 \\
\hline
\end{tabular}

simple transformation of fork rake (given by the manufacturer), $R$, to trail (used in the model), $c$, via some simple geometry as:

$$
c=\frac{r-R / \cos (\lambda)}{\tan (\lambda)}
$$

where $r=0.35 \mathrm{~m}$ for a $700 \mathrm{c}$ wheel. The remaining model parameters, $h$ and $a$, which define the vertical and horizontal position (respectively) of the centre of mass, $O$, must be determined by experiment and are addressed in Sections 3.1 and 3.2 respectively. While Table 1 shows the variation for the Trek 5200, the variation in head angle and rake (which are key determinants of stability) with frame size for some other popular road bikes is shown in Fig.3.
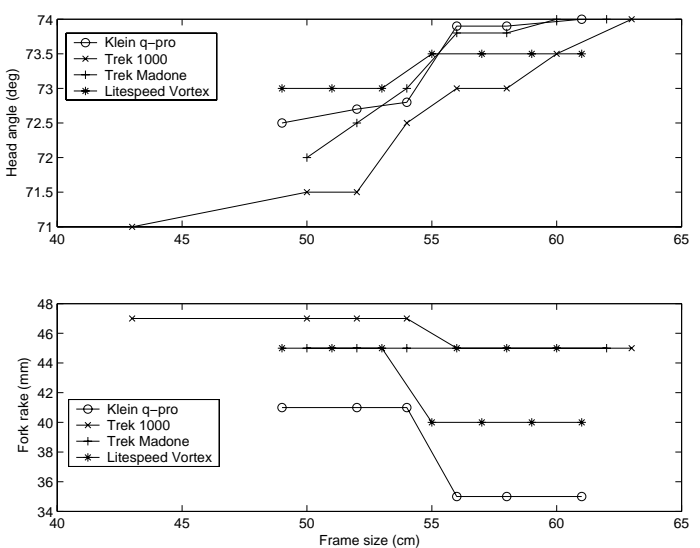

Figure 3: Typical head angle and rake variations.

\subsection{Determination of $a$}

The horizontal position of $O$ relative to the centre of the back wheel, $a$, may be determined by a see-saw balance to find the horizontal position of $O$. The setup is as shown in Fig.4. The bicycle, including rider, is moved back and forth in tiny increments until balance is achieved. This arrangement was also used to test the effect of an incline on $A$, with the following 


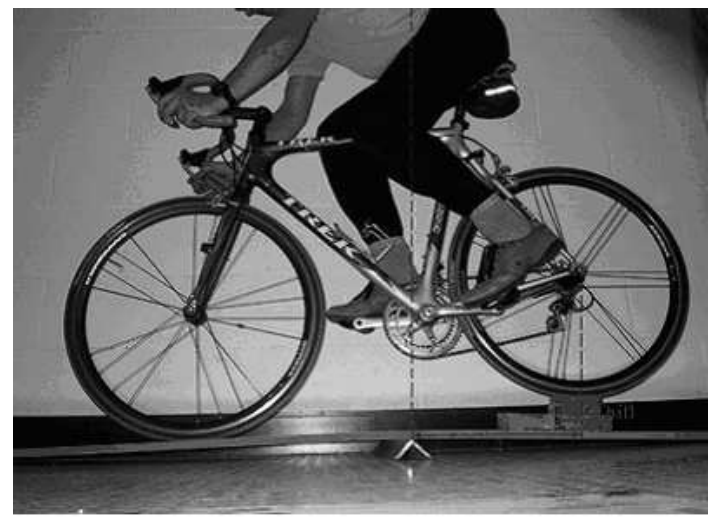

Figure 4: Experimental determination of $a$.

Table 2: Variation in $a$ with incline.

\begin{tabular}{|c|c|}
\hline Rear wheel height $(\mathrm{m})$ & $a(\mathrm{~m})$ \\
\hline 0 & 0.43 \\
\hline 0.045 & 0.46 \\
\hline 0.09 & 0.499 \\
\hline $0.09^{*}$ & 0.501 \\
\hline
\end{tabular}

results: The ${ }^{*}$, denotes a condition where, with an 'incline' of $0.09 \mathrm{~m}$, the rider leans forward to simulate a braking condition.

\subsection{Determination of $h$}

$h$ can also be determined by a slightly more complicated arrangement, as shown in Fig.5. The bicycle, including rider, is induced to act as a pendulum, by suspension of the bicycle from an overhead fixture. For small angles of swing, the period of the pendulum is:

$$
T=2 \pi \sqrt{l / g}
$$

where $l$ is the pendulum length (to the centre of mass). The movement of the bike/rider combination was measured by bluetooth-enabled MTx motion sensor from Xsens Motion Technologies. This allows the accurate 3 degree-of-freedom tracking of an object in motion. Fig. 6 shows a section of the output from the sensor for the degree of freedom most closely aligned with the pendulum motion. The average measured oscillation period, over three trials, is 2.622 secs. This accords well with (average) stopwatch measurements of zero-crossing time of 2.617. From the measurements in Fig.5 and (18), we can determine $h$ as 0.867 $\mathrm{m}$. Since the mass of the bicycle + rider is dominated by the rider $(80 \mathrm{~kg} \mathrm{Vs} 9 \mathrm{~kg}$ ), one would expect $O$ to be roughly at the centre of mass of the rider. The mean ratio of centre of mass to height in males has been de-

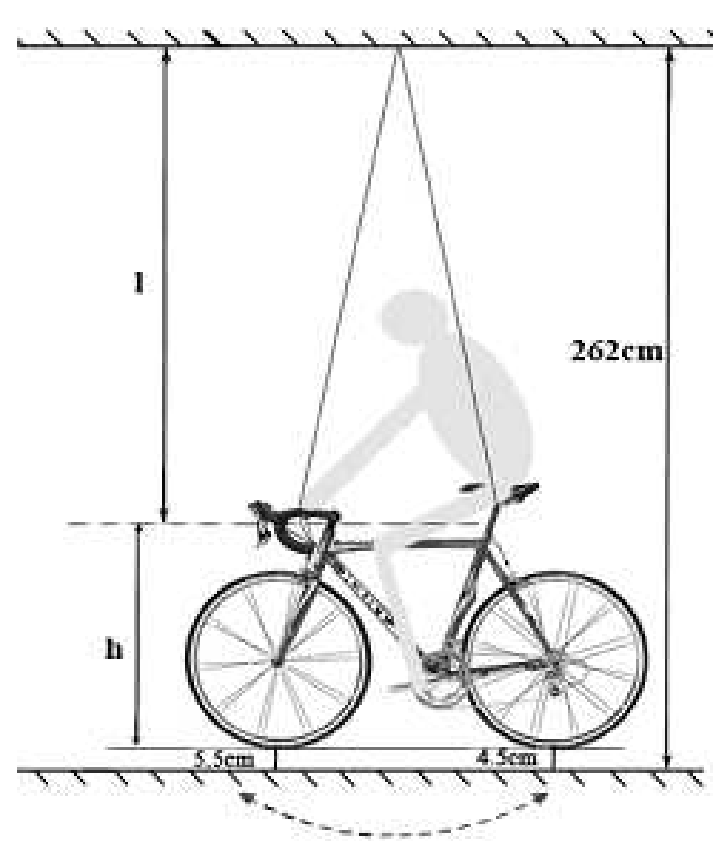

Figure 5: Experimental determination of $h$.

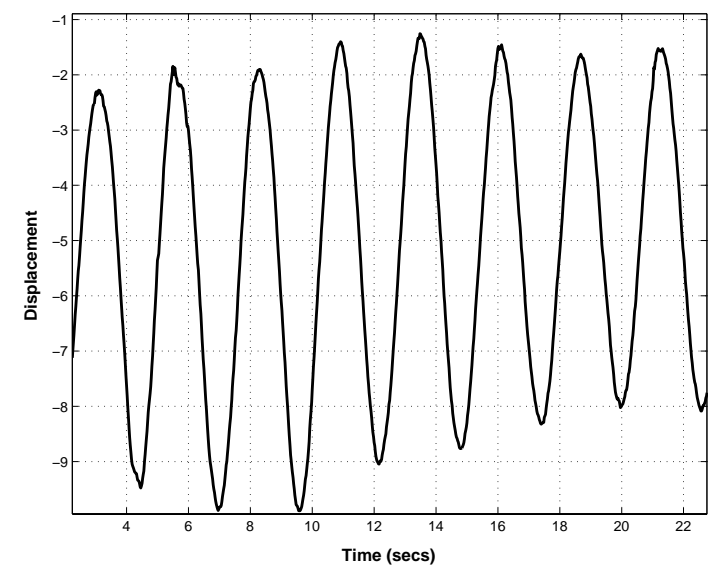

Figure 6: Pendulum period determination.

termined (Elert, 2006) as 0.565 , which would put $h$ roughly at the hip bone for the rider in question, consistent with the result obtained from the experimental determination. This provides a rough check on the experimental result.

\section{RESULTS}

In this section, we examine the stability of the bicycle model, for the parameters determined in Section 3, 
with variations in frame size and aspects which might relate to rider physique and position.

\subsection{Variation in Poles with Frame Size}

Using equation (14) and the data contained in Table 1, the pole variations may be plotted for a baseline condition of $a=0.43$ as shown in Fig.7. It can be noted
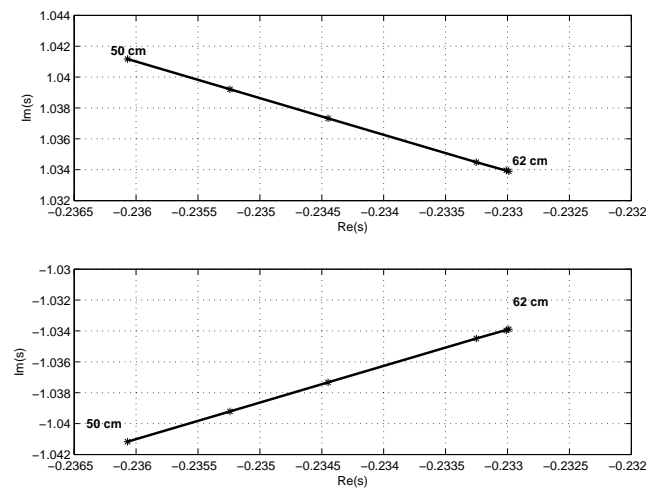

Figure 7: Pole variations with frame size.

that there is a perceptible movement of the poles towards the imaginary axis as the frame size increases. This suggests that, on the basis of frame geometry alone (not taking into account frame flexibility), there is a decrease in the stability of the bicycle with increasing frame size.

\subsection{Variation in Poles with Velocity}

Using equation (14), for a baseline condition of $a=$ 0.43 and a $60 \mathrm{~cm}$ frame, the variation in the poles with changes in velocity are computed as in Fig.8. It is clear that bicycle stability reduces as velocity increases. In the author's experience, the onset of wheel wobble typically occurs above $45 \mathrm{~km} / \mathrm{h}$. Note that the condition of basic bicycle self-stabilization in (7) is valid for the range of velocity considered in Fig.8.

\subsection{Rider Position Variations}

\subsubsection{Horizontal Position}

Again using equation (14), for a baseline condition of $V=45 \mathrm{~km} / \mathrm{h}$ (equivalent to $12.5 \mathrm{~m} / \mathrm{s}$ ) and a $60 \mathrm{~cm}$ frame, the pole variations with the relative forward position of the rider, $a$, can be evaluated, as shown in Fig.9. In this case, Fig.9 suggests that, as the rider moves more forwards on the bicycle, the stability of the bicycle increases. This would not have been the

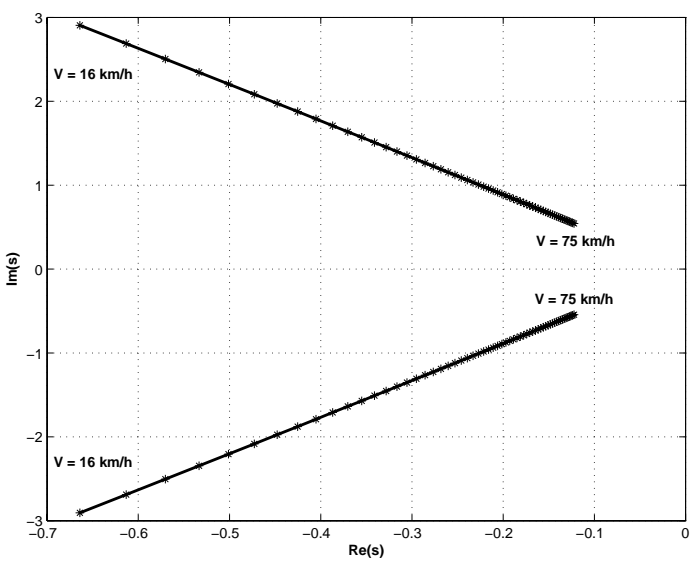

Figure 8: Pole variations with velocity.
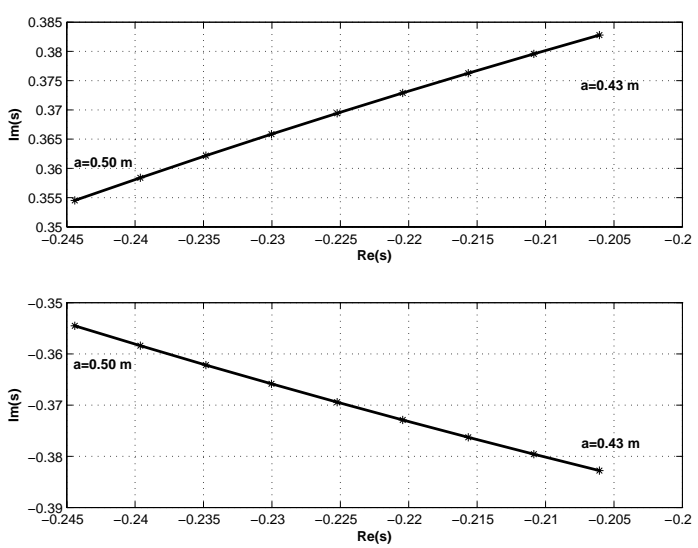

Figure 9: Pole variations with rider horiz. position.

impression of the author, but the result is supported by some anecdotal evidence presented in (Limebeer and Sharp, 2006), which documents a 'floating sensation' experienced by a motorcyclist on a record speed attempt while lying horizontal on the machine. This would, most certainly, have moved the centre of mass of the motorbike/rider combination to the rear, with a consequently relatively smaller value for $a$.

This result does not support the conclusion that the relative increase in $a$, due to the rider position moving forward under braking, causes any increased instability.

\subsubsection{Vertical Position}

Again using equation (14), for a baseline condition of $V=45 \mathrm{~km} / \mathrm{h}$, a $60 \mathrm{~cm}$ frame and $a$ returned to its nominal value of 0.43 , the pole variations with the relative vertical position of the rider, $h$, can be evaluated, as shown in Fig.10. From Fig.10, it is clear that increas- 

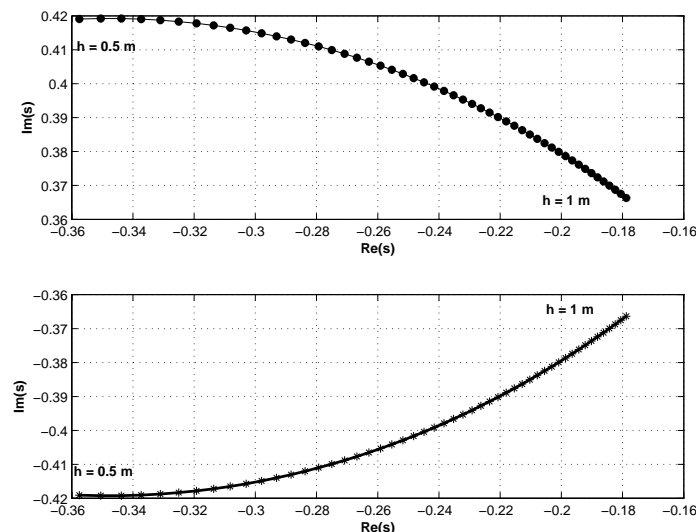

Figure 10: Pole variations with rider vertical position.

ing saddle height or a more upright position of the rider has a destabilizing effect on the bicycle, with a migration of the poles towards the imaginary axis.

\section{CONCLUSIONS}

Though the simple model in Section 2 omits some aspects of bicycle dynamics, such as frame compliance, mass of front fork assembly and rider intervention (via the torque input $T$ ), it can help to achieve an understanding of the broad effects that rider position and action has on bicycle stability. Though it is not possible to rely on absolute pole positions returned by the model, the relative pole movement under certain interventions can reveal the type of actions that can help to improve bicycle stability under certain conditions.

Clearly, road (racing) bicycles attempt to achieve a compromise between responsiveness and stability. This is largely dictated by frame geometry and trail (or fork rake). From the analysis in this paper, it appears that this compromise gives poorer stability for larger framed bicycles.

The impact of rider intervention via the torque input, $T$, deserves further examination. It is believed (Brandt, 2005) that attempting to reduce wheel wobble by rigidly holding the handlebars can, in fact, exaggerate it, due to the spring effect of the arms. One solution offered by an accomplished rider (who also has considerable experience in bicycle design) is to avoid holding the handlebars during fast descents (Brandt, 2006). While this is likely to alleviate stability problems due to frame shortening (as a result of braking) and exaggerated resonance via the arms, it may have it's own particular perils! The effect of rider steering action could be included as a feedback term in the model, though the parameters of such a subsystem may not be trivial to determine.

Further work should also examine the effect of frame compliance, since this is thought to be an important factor leading to wheel wobble and is likely to be more pronounced in bicycles with larger frames. However, some effects which result from component compliance can be examined within the current model structure. In particular, braking (with most of the braking effect coming from the front wheel) is likely to lead to some shortening of the wheelbase, due to flexibility in the (carbon) forks. This could cause a reduction in both $b$ (the wheelbase) and $R$ (the fork rake). The model predicts that a reduction in both these values would have a destabilizing effect, which could more than offset any stabilizing effect resulting from a movement forward in the centre of mass (under downhill braking), examined in Section 4.3.1.

\section{ACKNOWLEDGEMENTS}

The authors are grateful to Denis Buckley, John Maloco and Dr. Tomás Ward of the Dept. of Electronic Eng. ay NUI Maynooth for their contribution to the experimental measurements of Section 3.1 and 3.2.

\section{REFERENCES}

Astrom, K., Klein, R., and Lennartsson, A. (2005). Bicycle dynamics and control - adapted bicycles for education and research. IEEE Control Systems Mag., 25:26-47.

Beghi, A. and Frezza, R. (2006). Advances in motorcycle design and control. IEEE Control Systems Mag., 26:32-33.

Brandt, J. (2005). Shimmy or speed wobble. http://www.sheldonbrown.com/brandt/shimmy.html.

Brandt, J. (2006). Speed wobble. Private correspondence.

Elert, G. (2006). Centre of mass of a human. In The Physics Factbook. http://hypertextbook.com/facts/2006/centerofmass.shtml.

Hauser, J. and Saccon, A. (2006). Motorcycle modelling for high-performance manouvering. IEEE Control Systems Mag., 26:89-105.

Klein, R. (1989). Using bicycles to teach system dynamics. IEEE Control Systems Mag., 9:4.

Limebeer, D. and Sharp, R. (2006). Bicycles, motorcycles and models - single track vehicle modelling and control. IEEE Control Systems Mag., 26:34-61.

Rankine, W. (1869). On the dynamical properties of the motion of velocipedes. Engineer, 28:79-175. 